\title{
Structure of a media co-occurrence network
}

\author{
V.A. $\operatorname{Traag}^{1}$, R. Reinanda ${ }^{2}$, and G. van Klinken ${ }^{1}$ \\ ${ }^{1}$ KITLV, Leiden, the Netherlands \\ 2 Faculty of Science, University of Amsterdam, Amsterdam, the Netherlands
}

\begin{abstract}
Social networks have been of much interest in recent years. We here focus on a network structure derived from co-occurrences of people in traditional newspaper media. We find three clear deviations from what can be expected in a random graph. First, the average degree in the empirical network is much lower than expected, and the average weight of a link much higher than expected. Secondly, high degree nodes attract disproportionately much weight. Thirdly, relatively much of the weight seems to concentrate between high degree nodes. We believe this can be explained by the fact that most people tend to co-occur repeatedly with the same people. We create a model that replicates these observations qualitatively based on two self-reinforcing processes: (1) more frequently occurring persons are more likely to occur again; and (2) if two people co-occur frequently, they are more likely to co-occur again. This suggest that the media tends to focus on people that are already in the news, and that they reinforce existing co-occurrences.
\end{abstract}

\section{Introduction}

Complex networks have been a prominent research topic for the past decade. One of the reasons is that complex networks appear in a multitude of scientific disciplines, varying from neurology [6]18, ecology [13 16] to international relations 92214] and human mobility 15/29] providing a unified theoretical framework for analysis. Although many properties seem to be (nearly) universal (e.g. degree distribution, clustering) 2, there are also some noteworthy differences between different types of networks (e.g. assortativity, weak links) [27/17/1].

Social networks can nowadays be relatively easily scraped from online services such as Facebook or Twitter 8 83211. In addition, traditional media (i.e. newspapers) are also increasingly being digitised. Whereas online social media are open to the general public, and the large masses use them intensively, traditional media are biased towards the more influential members of society. Therefore, we might learn something about the elite of a society by studying how they appear in the newspapers. In this study, we focus on who co-occurs with whom. We aim to understand the structure of the co-occurrences. Do frequently occurring people co-occur mostly with each other, or not? How strongly do frequently occurring people connect to each other?

Given the possibilities of using media co-occurrence reports for constructing social networks, there have been surprisingly few earlier studies of media cooccurrence networks 30 28 34 19]. The analyses were relatively succinct, and 
showed that such networks were both scale-free and a small world, properties we also find here. However, the scale-free and small world nature of these cooccurrence networks can be expected, and also show up in a randomised graph. In fact, various common properties are quite close to what can be expected in a randomised graph. Nonetheless, three deviations from the random graph stand out. First of all, the average degree is much higher than expected, while the average weight is much lower than expected. Secondly, high degree nodes attract disproportionately much weight. Thirdly, much of the weight is concentrated in between high degree nodes.

These observations suggest that people repeatedly occur with the same people, or at least more so than expected at random. To explain this, we create a model that concentrates more of the co-occurrences in fewer people, thus explaining this deviation. The model consists of only two simple ingredients: (1) more frequently occurring people tend to occur more frequently in the future; and (2) people that co-occur more frequently tend to co-occur more frequently in the future. In addition, high degree nodes are more likely to occur with already existing neighbours.

\section{Data \& Network}

We use newspaper articles to construct a social network. The idea is that people are linked if they co-occur in the same sentence. We use two corpora in our current study: (1) a corpus from an Indonesian news service called Joy ${ }^{3}$, and (2) a corpus from the New York Time ${ }^{4}$ (NYT). The Joyo dataset covers roughly 2004-2012 and contains 140263 articles, while the NYT dataset covers 19871988 and contains 210645 articles. The Joyo dataset is a selection of political news (in English) from both domestic and foreign sources that is relevant to the politics of Indonesia, while the NYT is a complete corpus of all the articles of that newspaper. We scan the whole text and automatically identify entities by using a technique known as named entity recognition (NER) 12. The technique automatically identifies different entities, and classifies them into three distinct categories: persons, organisations and locations. Although it is not perfect, the error rate tends to be relatively low [12].

We have only included persons in our media co-occurrence network, and only people that occurred in more articles than on average. We thereby exclude people that only appear quite infrequently, which are presumably less influential, and thus less of interest. Although this skews the results more towards people that appear more prominently in the media, it still includes many less prominent people. Once all persons have been identified, we have to disambiguate them. There are generally two types of errors that can be made with names 23: (1) a single name corresponds to two different persons (e.g. "Bush" can refer to the $43^{\text {rd }}$ or $41^{\text {st }}$ US president); and (2) two different names refer to the same person

\footnotetext{
3 http://www . joyonews.org

4 See https://catalog.ldc.upenn.edu/LDC2008T19 for the corpus. We only used the first two years of the dataset.
} 


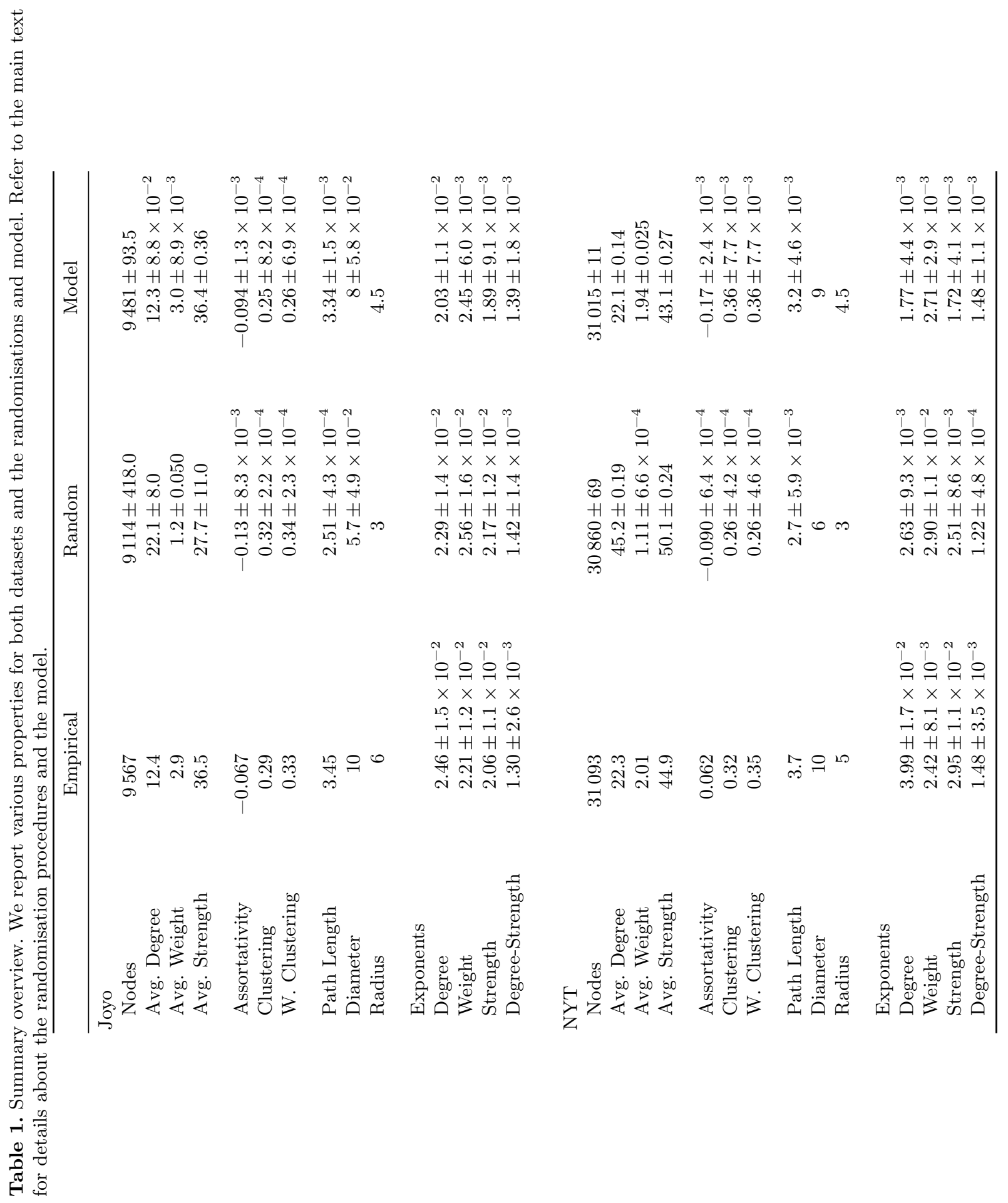


("President Clinton" or "Bill Clinton" both refer to the $42^{\text {nd }}$ US president). The second problem appears much more prominent than the first problem in our corpus, as people are generally referred to in many different ways in journalistic prose (including or not positions, titles, initials, maiden names, etc...).

We disambiguated these names by using a combination of similarity measures based on Wikipedia matching, string similarity and network similarity (using Jaccard similarity). The more prominent people often have a Wikipedia page, and various spelling variants are redirected to the same entity (e.g. "President Clinton" and "Bill Clinton" both redirect to the same Wikipedia page). Each similarity is normalised to fall between 0 and 1 (with 1 being identical), which we threshold at 0.75 , such that we only take it into account if the similarity is at least 0.75 . We then take the average of the similarities that are higher than 0.75 (which is then also at least 0.75). We then find clusters of names such that each cluster has an average internal similarity of 0.85 (all similarity measures are between 0 and 1), using a technique called the Constant Potts Model [31.

Once the names have been disambiguated, we create a link for all the unique names in a sentence (i.e. repeated names have no effect). We do this for every sentence, and simply count in how many sentences such a co-occurrence was observed. We take only the largest connected component of the network, and this constitutes the media co-occurrence network we analyse in this paper.

Of course, what co-occurrence exactly implies is not always clear: two people might be mentioned together for example because they collaborate, or because they are contestants in an election. A co-occurrence might not coincide with any one single definition of a "relationship" in the sociological sense [20. Hence, we cannot say if two people that co-occur have any more significant relationship: do they know each other? Have they ever communicated? Have they met face to face? Are they close friends? Sworn enemies? We simply cannot tell. This is essential to bear in mind when drawing any conclusions: the network is based on co-occurrence, not on "actual relationships".

\section{$3 \quad$ Results}

We denote the undirected network by $G=(V, E)$ where $V=\{1, \ldots, n\}$ is the node set, representing the people, and $E \subseteq V \times V$ constitutes the edge set with $m=|E|$ edges, representing the co-occurrences between people. Hence, if node $i \in V$ and node $j \in V$ co-occurred in some sentence, then there is an edge $(i j) \in E$. Each edge has a weight associated to it, which represents the number of times the two nodes $i$ and $j$ have co-occurred, which we denote by $w_{i j}$. Finally, the adjacency matrix is denoted by $A$, such that $A_{i j}=1$ if there is an edge between node $i$ and $j$ and zero otherwise. Since the network is undirected, we have that $A_{i j}=A_{j i}$ and $w_{i j}=w_{j i}$. Notice that this network is a projection of a bipartite network of people and sentences. Let us denote by $B$ the bipartite adjacency matrix, so that $B_{i s}=1$ if person $i$ occurs in sentence $s$. Then $w_{i j}=\sum_{s} B_{i s} B_{j s}$ and $A_{i j}=1$ if $w_{i j}>0$ and $A_{i j}=0$ otherwise. In 


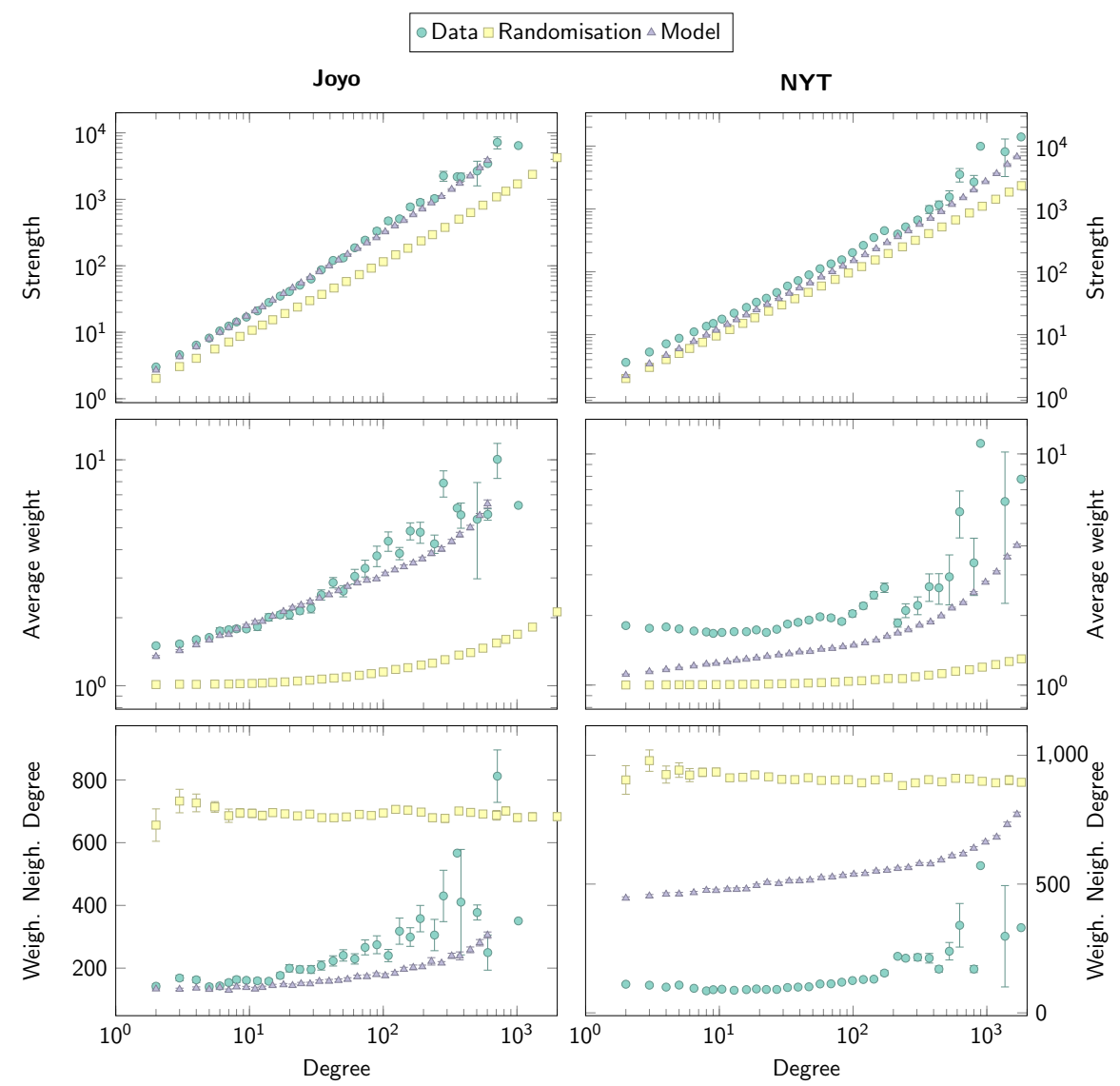

Fig. 1. Properties. The first column shows the properties for Joyo, the second for NYT. This clearly shows that the strength increases faster than expected (first row), which is even more clear when looking at the average strength (second row). The model however, captures quite well this increase, especially for Joyo. The weighted neighbour degree increases, whereas this remains nearly constant for the randomisation. The model again, shows a relatively similar increase, especially for Joyo.

other words, $w=B B^{T}$. We denote the degree of node $i$ by $k_{i}=\sum_{j} A_{i j}$ and the strength by $s_{i}=\sum_{j} A_{i j} w_{i j}$.

We compare our results to a bipartite randomisation of the network. Formally, let $P$ be a list of persons and $S$ a list of sentences, such that $\left(P_{e} S_{e}\right)$ is a bipartite edge, so that the length of the list equals the number of bipartite edges. If $\sigma$ is a random permutation, then $\left(P_{\sigma_{e}} S_{e}\right)$ for all $e$ are the randomized edges. Simply put, this randomisation takes the list of occurrences of people in sentences and shuffles the complete edge list, so that people occur in a random 
sentence. This preserves the number of times somebody occurs in a sentence, and preserves the number of people that occur in a sentence. We then take a projection of this network as we did for the empirical observations. In other words, $\hat{w}=\hat{B} \hat{B}^{T}$ and $\hat{A}_{i j}=1$ if $\hat{w}_{i j}>0$, where $\hat{B}_{P_{\sigma_{e}} S_{e}}=1$ for all $e$ and zero otherwise. We create a 100 different randomisations and use it to compare to our empirical observations.

The Joyo network has $n=9467$ nodes, an average degree of $\left\langle k_{i}\right\rangle \approx 12.22$ and with an average weight per edge of $\left\langle w_{i j}\right\rangle \approx 2.95$ the average strength is $\left\langle s_{i}\right\rangle=\left\langle k_{i}\right\rangle\left\langle w_{i j}\right\rangle \approx 36.07$. So, in Joyo, a person co-occurs on average about 3 times with 12 other people, so in total about 36 times. Based on the randomisation, we would expect roughly 22 people to occur about 1.2 times, giving a total strength of around 28 . Hence, people co-occur roughly 2.5 times more often with the same people as expected, and co-occur with almost 2 times less people. The NYT corpus contains $n=31093$ nodes and has an average degree of about $\left\langle k_{i}\right\rangle \approx 22.35$. The average weight $\left\langle w_{i j}\right\rangle \approx 2.01$, which gives an average strength of about $\left\langle s_{i}\right\rangle \approx 44.91$. In summary, a person in the NYT co-occurs about 2 times with about 22 different people. Similar as for Joyo, based on the randomisation, we would expect around 45 people to occur about 1.1 times, giving a total strength of about 50 . So, in NYT people co-occur almost 2 times more frequently with the same people, with roughly 2 times fewer people. We provide an overview of some of the key statistics in Table 1.

The degree, strength and weight are all heterogeneously distributed, as frequently observed in complex networks. They follow approximately powerlaws [7] in both networks, where we use MLE techniques for estimating the powerlaws. The degree and strength in the Joyo corpus is more broadly distributed compared to the NYT corpus, but the weight is distributed similarly. However, these distributions are also expected to be heterogeneous based on the randomisation. Although there are some deviations, they seem mainly due to the lower average empirical degree and the higher average empirical weight.

The network shows signs of a small world network. The average path length is relatively low, while the clustering is relatively high. Again, this does not deviate much from what is expected at random. The (weighted) clustering is almost the same, and the path length is even slightly longer than expected at random. The longer path length is probably due to the lower average degree. With fewer neighbours on average, there are fewer possibilities for paths, thus leading to somewhat longer paths.

We find that the strength scales superlinearly with the degree as $s_{i} \sim k_{i}^{\beta}$, with an exponent of $\beta \approx 1.30$ for Joyo and $\beta \approx 1.48$ for NYT. This means that high degree nodes attract more weight and low degree nodes less weight. In this context, people that co-occur with many other people, also tend to co-occur more often. A similar superlinear scaling was found in transportation and technological networks $33[26] 5$. This contrasts with for example mobile phones where there is a sublinear growth [25], suggesting that people who call many people, do so less frequently than people who call few people. This is quite different from what is expected at random, especially when looking at this from the perspective of the 
average weight $s_{i} / k_{i}$, which increases for large degree $k_{i}$ empirically, but which increases only very slightly in the randomisation. We have plotted the behaviour of the average weight and the strength in Fig. 1 .

The average weighted neighbour degree [4] increases with larger degree. This implies that high degree nodes connect relatively stronger to other high degree nodes. For the NYT the weighted neighbour degree starts to increases more clearly for a degree larger than about 200. This suggest that relatively much weight is in between high degree nodes. See Fig. 1 for plots of the weighted neighbour degree. The ordinary neighbour degree decreases for Joyo, as evidenced by the negative assortativity 24, whereas this increases for NYT (Table 1), pointing out a difference between the two datasets.

\subsection{Model}

Three phenomena deviate from what can be expected from a random graph. First, the degree is higher than expected and the weight is lower than expected. Secondly, high degree nodes attract disproportionately much weight. Thirdly, much of the weight is between the hubs. These observations suggest that people tend to co-occur repeatedly with the same people. We therefore introduce a very simple stylistic model that is able to reproduce most of the observations in the empirical network qualitatively. The model consists of two key ingredients: (1) more frequently occurring people have a higher probability of occurring; and (2) two more frequently co-occurring people have a higher probability of co-occurring.

More specifically, we employ the following procedure. We start out with an empty graph. Each time step, we draw a random sentence $s$, with degree $k_{s}$ (i.e. the number of people occurring in a sentence) drawn from the empirical sentence degree distribution. We then choose $k_{s}$ nodes in the following way. With probability $q$ we introduce a new person into the graph, which is chosen so that the expected number of nodes equals the number of nodes $n$ in the empirical graph. That is, if $p_{k}$ is the probability a sentence has degree $k$, then the total expected number of nodes occurring in sentences will be $n_{s} \sum_{k} k p_{k}$, with $n_{s}$ the number of sentences. So, if $q=\frac{n}{n_{s} \sum_{k} k p_{k}}$ we generate on average about $n$ nodes.

If we don't introduce a new node, we pick a random node $i$ in the sentence. Then with probability $\left(k_{i}+1\right)^{-\beta}$ we choose an already existing node, where $k_{i}$ is the degree of node $i$ and $\beta$ a tunable parameter. The probability a node is selected is proportional to its degree, so that $\operatorname{Pr}($ choose $j)=k_{j} / \sum_{l} k_{l}$. If we don't pick an existing node, we choose a random neighbour of $i$ with probability proportional to the weight, so that $\operatorname{Pr}($ choose $j \mid i)=w_{i j} / \sum_{k} w_{i k}$. After we picked all $k_{s}$ nodes, we create an edge for all combinations of persons in the sentence. If an edge already exists, we increase its weight.

The node sampling is very similar to the preferential attachment model from Barabási and Albert [3] and similar models [10, as nodes that have more links are more likely to receive additional links. However, our model differs in several important ways from the model by Barabási and Albert [3]. First of all, it tends 
to generate a superlinear scaling of the strength with the degree. Secondly, it generates a much higher clustering coefficient. This latter effect is mainly a result of sampling neighbours, which relates to triadic closure, which has also been used in other models [21.

Besides preferential attachment, our model also has a counter tendency. Higher degree nodes are increasingly more likely to co-occur with already existing neighbours. The idea behind the scaling $\left(k_{i}+1\right)^{-\beta}$ is based on the idea that higher degree nodes have a higher than linear strength. In other words, hubs are more likely to repeatedly co-occur with their neighbours, more so than on average.

A crude argument shows that indeed this model should result in superlinear scaling of the strength. Consider $k_{i}\left(s_{i}\right)$, the degree of node $i$ as a function of the strength $s_{i}$, and suppose that all sentences have only degree 2 . Every time we add a co-occurrence for $i, s_{i}$ increases, although $k_{i}$ does not necessarily increase. Now if $i$ was the first node to be chosen, it will get a new neighbour with probability $\left(k_{i}+1\right)^{-\beta}$. If $i$ was the second node to be chosen, it implies it is chosen by another node. The probability that this node selected a new neighbour (which by definition then is node $i$, since we already know it was chosen) is then $\left(k_{j}+1\right)^{-\beta}$ given that node $j$ was chosen first. But the probability that node $j$ was chosen first is proportional to $k_{j}$. Then the probability $i$ gets a new neighbour if $s_{i}$ increases is

$$
\left(k_{i}+1\right)^{-\beta}+\sum_{j} \frac{k_{j}}{\sum_{l} k_{l}}\left(k_{j}+1\right)^{-\beta} .
$$

Taking a mean-field approach, we approximate $k_{i} \approx\langle k\rangle$, and simply write $k=$ $\langle k\rangle$ for ease of writing, we obtain that

$$
\Delta k=(k+1)^{-\beta}+\sum_{j} \frac{k}{\sum_{l} k}(k+1)^{-\beta}=2(k+1)^{-\beta} .
$$

Taking then the approximation that $\partial k / \partial s \approx \Delta k$, we obtain the solution that $s \sim k^{1+\beta}$ so that the strength increases superlinearly with $k$. This is of course a rather crude argument, but it nonetheless shows that using this approach we should indeed expect a superlinear scaling of the strength with the degree.

This contrasts with using a constant probability for choosing a new neighbour. In that case, essentially each time that the strength increases, the probability that the degree increases is a fixed probability $\rho$. This then results in $k \sim \rho s$, showing only linear scaling.

Additionally, this model has the tendency to create a relatively high clustering coefficient. Every time that a new sentence is introduced with $k_{s}$ persons, all these $k_{s}$ persons will be connected amongst each other, creating small cliques. In addition, these cliques are also reinforced by the mechanism of adding neighbours to sentences. Interestingly, despite such reinforcement, the model still generates a dissortative structure [24]. Although this is congruent with Joyo, it contrasts with the assortative structure for NYT.

In order to estimate the parameter $\beta$, we compare the average degree and average weight to the empirically observed values. That is, for each parameter 
value $\beta$, we compare the average degree $\left\langle\hat{k}_{i}\right\rangle$ and average weight $\left\langle\hat{w}_{i j}\right\rangle$ of the model, and compare that to the average degree $\left\langle k_{i}\right\rangle$ and average weight $\left\langle w_{i j}\right\rangle$ of the empirical network. We use a simple squared error for the fitting,

$$
E(\beta)=\left(\left\langle\hat{k}_{i}\right\rangle-\left\langle k_{i}\right\rangle\right)^{2}+\left(\left\langle\hat{w}_{i j}\right\rangle-\left\langle w_{i j}\right\rangle\right)^{2}
$$

We used 100 replications for each parameter value. The best parameter fit differs quite a bit between Joyo and NYT. For Joyo we find an optimal parameter of $\beta \approx 0.46$, while for NYT we find $\beta \approx 0.22$. See Fig. 2 for the fitting of the parameter.

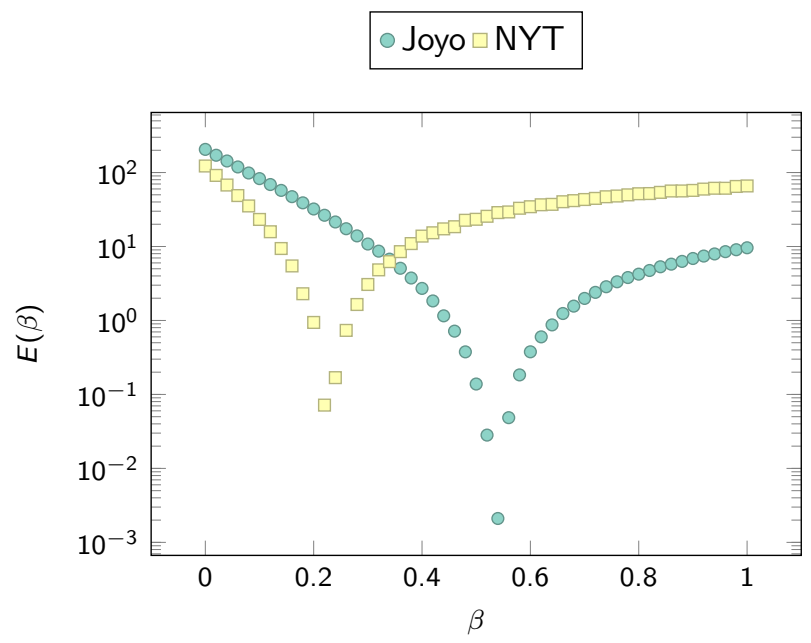

Fig. 2. Model fit. The fit of the parameter $\beta$ in the model. The error $E(\beta)$ it taken with respect to the average degree and the average weight.

As expected, we can fit the empirically observed average degree and weight very well. Additionally, we indeed observe a very similar increase in the average weight for high degree nodes, as was already argued above. Finally, the model also shows an increase in the average weighted neighbour degree, similar as empirically. Nonetheless, although the average degree is quite well fit for NYT, the distribution of the degree is more broader, leading to a higher average weighted neighour degree. Nonetheless, both the model and the empirical network show an increase. The fit of the model for Joyo is especially striking in Fig. 1. Perhaps this is because Joyo has a more particular focus on politics, while the NYT includes also other subjects such as culture, arts and sports. 


\section{Conclusion}

In this paper we analysed two networks based on the co-occurrence of people in newspapers. We have analysed various properties of this network, and whereas many properties are in line with what could be expected from such a co-occurrence network, a few deviations stand out. First, people occur with fewer people than expected and more often with those people than expected. Secondly, high degree nodes attract disproportionately much weight, so that the hubs co-occur much more often than their degree justifies. Third, much of the weight concentrates between these hubs.

This suggests that people repeatedly co-occur with the same people. We constructed a model that tries to reproduce these observations. It is based on two simple processes: (1) people that occur in the media are more likely to occur again; (2) two people that co-occur are more likely to co-occur again. Moreover, people with a higher degree co-occur more often with people with whom they already co-occur. This seems to explain the observations quite well, although some deviations remain.

There are some clear differences between the Joyo and the NYT corpus. Whether this is reflective of differences between Indonesia and the US, or the more politically oriented corpus of Joyo, or a difference in time periods, is difficult to ascertain. Further analysis and comparison of these networks should provide more insight.

\section{Acknowledgements}

VT would like to thank Fabien Tarissan for interesting comments and remarks on an earlier version of this manuscript. This research is funded by the Royal Netherlands Academy of Arts and Sciences (KNAW) through its eHumanities project 5

\section{References}

1. Amaral, L.A.N., Scala, A., Barthélémy, M., Stanley, H.E.: Classes of small-world networks. Proc. Natl. Acad. Sci. USA 97(21), 11149-11152 (2000)

2. Barabási, A.L.: Scale-free networks: A decade and beyond. Science 325(5939), 412413 (2009)

3. Barabási, A.L., Albert, R.: Emergence of scaling in random networks. Science 286(5439), 509-512 (1999)

4. Barrat, A., Barthélemy, M., Pastor-Satorras, R., Vespignani, A.: The architecture of complex weighted networks. Proc. Natl. Acad. Sci. USA 101(11), 3747-3752 (2004)

5. Barthélemy, M., Barrat, A., Pastor-Satorras, R., Vespignani, A.: Characterization and modeling of weighted networks. Physica A 346(1-2), 34-43 (2005)

5 http://www.ehumanities.nl/computational-humanities/

elite-network-shifts/ 
6. Bullmore, E., Sporns, O.: Complex brain networks: graph theoretical analysis of structural and functional systems. Nat. Rev. Neurosci. 10(3), 186-98 (2009)

7. Clauset, A., Shalizi, C.R., Newman, M.E.J.: Power-law distributions in empirical data. SIAM Rev. Soc. Ind. Appl. Math. 51(4), 661-703 (2009)

8. Corten, R.: Composition and structure of a large online social network in the netherlands. PLoS ONE 7(4), e34760 (2012)

9. Cranmer, S.J., Menninga, E.J., Mucha, P.J.: Kantian fractionalization predicts the conflict propensity of the international system. arXiv:1402.0126 [physics] (2014)

10. Dorogovtsev, S.N., Mendes, J.F.F., Samukhin, a.N.: Structure of growing networks with preferential linking. Phys. Rev. Lett. 85(21), 4633-4636 (2000)

11. Ferrara, E.: A large-scale community structure analysis in facebook. EPJ Data Sci. 1(1), 9 (2012)

12. Finkel, J.R., Grenager, T., Manning, C.: Incorporating non-local information into information extraction systems by gibbs sampling. In: Proceedings of the 43rd Annual Meeting on Association for Computational Linguistics. p. 363-370. Association for Computational Linguistics, Stroudsburg, PA, USA (2005)

13. Garlaschelli, D., Caldarelli, G., Pietronero, L.: Universal scaling relations in food webs. Nature 423(6936), 165-8 (2003)

14. Garlaschelli, D., Loffredo, M.I.: Structure and evolution of the world trade network. Physica A 355(1), 138-144 (2005)

15. González, M.C., Hidalgo, C.A., Barabási, A.L.: Understanding individual human mobility patterns. Nature 453(7196), 779-82 (2008)

16. Guimerà, R., Stouffer, D.B., Sales-Pardo, M., Leicht, E.A., Newman, M.E.J., Amaral, L.A.N.: Origin of compartmentalization in food webs. Ecology 91(10), 2941-2951 (2010)

17. Guimerà, R., Sales-Pardo, M., Amaral, L.A.N.: Classes of complex networks defined by role-to-role connectivity profiles. Nat. Phys. 3(1), 63-69 (2007)

18. Hagmann, P., Cammoun, L., Gigandet, X., Meuli, R., Honey, C.J., Wedeen, V.J., Sporns, O.: Mapping the structural core of human cerebral cortex. PLoS Biol. 6(7), e159 (2008)

19. Joshi, D., Gatica-Perez, D.: Discovering groups of people in google news. In: Proceedings of the 1st ACM International Workshop on Human-centered Multimedia. p. 55-64. HCM '06, ACM, New York, NY, USA (2006)

20. Knoke, D., Yang, S.: Social Network Analysis. No. 154 in Quantitative Applications in the Social Sciences, SAGE Publications, Inc, Cambridge, Mass, 2nd edition edn. (2007)

21. Kumpula, J.M., Onnela, J.p., Saramäki, J., Kaski, K., Kertész, J.: Emergence of Communities in Weighted Networks. Phys. Rev. Lett. 99(22), 228701 (2007)

22. Maoz, Z., Terris, L.G., Kuperman, R.D., Talmud, I.: What is the enemy of my enemy? causes and consequences of imbalanced international relations, 1816-2001. J. Politic. 69(01), 100-115 (2008)

23. Milne, D., Witten, I.H.: Learning to link with wikipedia. In: Proceedings of the 17th ACM Conference on Information and Knowledge Management. p. 509-518. CIKM '08, ACM, New York, NY, USA (2008)

24. Newman, M.E.J.: Assortative Mixing in Networks. Phys. Rev. Lett. 89(20), 208701 (2002)

25. Onnela, J.P., Saramäki, J., Hyvönen, J., Szabó, G., de Menezes, M.A., Kaski, K., Barabási, A.L., Kertész, J.: Analysis of a large-scale weighted network of one-to-one human communication. New. J. Phys. 9(6), 179-179 (2007) 
26. Ou, Q., Jin, Y.D., Zhou, T., Wang, B.H., Yin, B.Q.: Power-law strength-degree correlation from resource-allocation dynamics on weighted networks. Phys. Rev. E 75(2), 021102 (2007)

27. Petri, G., Scolamiero, M., Donato, I., Vaccarino, F.: Topological strata of weighted complex networks. PLoS ONE 8(6), e66506 (2013)

28. Pouliquen, B., Tanev, H., Atkinson, M.: Extracting and learning social networks out of multilingual news. In: Social Networks and application tools (2008)

29. Simini, F., González, M.C., Maritan, A., Barabási, A.L.: A universal model for mobility and migration patterns. Nature 484(7392), 96-100 (2012)

30. Steinberger, R., Pouliquen, B.: Cross-lingual named entity recognition. Ling. Inv. 30(1), 135-162 (2007)

31. Traag, V.A., Van Dooren, P., Nesterov, Y.: Narrow scope for resolution-limit-free community detection. Phys. Rev. E 84(1), 016114 (2011)

32. Traud, A.L., Mucha, P.J., Porter, M.A.: Social structure of facebook networks. Physica A 391(16), 4165-4180 (2012)

33. Wang, W.X., Wang, B.H., Hu, B., Yan, G., Ou, Q.: General dynamics of topology and traffic on weighted technological networks. Phys. Rev. Lett. 94(18), 188702 (2005)

34. Özgür, A., Bingol, H.: Social network of co-occurrence in news articles. In: Aykanat, C., Dayar, T., Korpeoglu, I. (eds.) Computer and Information Sciences - ISCIS 2004, pp. 688-695. No. 3280 in Lecture Notes in Computer Science, Springer Verlag, Heidelberg (2004) 